\title{
Non-accidental trauma increases length of stay and mortality in pediatric trauma
}

\author{
J. K. Livingston ${ }^{1} \cdot$ A. Grigorian ${ }^{1} \cdot$ C. M. Kuza ${ }^{2} \cdot$ M. Lekawa ${ }^{1} \cdot$ N. Bernal ${ }^{1} \cdot$ A. Allen ${ }^{1} \cdot$ J. Nahmias $^{1}$
}

Accepted: 29 April 2019/ Published online: 10 May 2019

○) Springer-Verlag GmbH Germany, part of Springer Nature 2019

\begin{abstract}
Purpose More than half a million children experience non-accidental trauma (NAT) annually. Historically, NAT has been associated with an increased hospital length of stay (LOS). We hypothesized that in pediatric trauma patients, NAT is associated with longer hospital LOS, independent of injury severity, compared to accidental trauma (AT).

Methods The Pediatric Trauma Quality Improvement Program (2014-2016) was queried for patients aged 1-16 years. Patients were stratified into two groups: AT and NAT. The median LOS for the entire cohort was identified and used in a multivariable logistic regression analysis.

Results From 93,089 pediatric trauma patients, $417(<0.1 \%)$ were involved in NAT. Patients with NAT had a lower median age (3 vs. 9 years, $p<0.001)$ and higher median injury severity score $(10$ vs. $5, p<0.001)$, compared to patients with AT. After controlling for covariates, patients with NAT were associated with a longer hospital LOS ( $\geq 2$ days), compared to those with AT $(\mathrm{OR}=4.99 \mathrm{CI}=3.55-7.01, p<0.001)$. In comparison to AT, NAT was also associated with a higher mortality rate $(10.3 \%$ vs. $0.8 \%, p<0.001)$.

Conclusion Pediatric patients presenting after NAT have a prolonged hospital and ICU LOS, even after adjusting for injury severity. Furthermore, pediatric victims of NAT had a higher mortality rate compared to those presenting after AT.
\end{abstract}

Keywords Non-accidental trauma $\cdot$ Length of stay $\cdot$ Mortality $\cdot$ Child abuse

\section{Introduction}

In 2016, Child Protective Services (CPS) received an estimated 4.1 million referrals for suspected non-accidental trauma (NAT) and neglect in pediatric patients, with approximately 670,000 confirmed cases. While neglect represents the most common form of child abuse (75\%), nearly $20 \%$ of victims experience physical abuse. NAT represents a leading cause of childhood traumatic injury, including serious injuries such as severe traumatic brain injury (TBI), major torso trauma, and traumatic injuries to the extremities. Furthermore, NAT accounts for an estimated 1750 deaths annually in the United States [1,2]. Although there have been

$\triangle$ J. K. Livingston

jklivin1@uci.edu

1 Department of Surgery, University of California Irvine, 101 The City Dr S, Orange, CA 92868, USA

2 Department of Anesthesiology, Keck School of Medicine of the University of Southern California, 1450 San Pablo Street, Suite 3600, Los Angeles, CA 90033, USA increasing efforts to implement child protective services and screening methods to detect NAT, the number of admissions and deaths from NAT have not decreased since 1970 [3]. In fact, the number of NATs increased from 2011 to 2014, mainly due to improvements in screening and reporting [4]. However, many cases of NAT are still missed [4], with reports of up to $20 \%$ of NAT cases either not recognized [5], or diagnosed late [3]. Although many hospitals have protocols in place in attempts to identify NAT early, a recent survey of U.S. hospitals demonstrated significant variability in NAT screening practices [6].

When NAT is suspected, an extensive investigation, entailing an exhaustive screening process, interviews with family, witnesses, and/or caregivers, and a risk/safety assessment is performed before a decision deeming the trauma to be a NAT is made. The inpatient hospitalization of a child suspected of abuse or neglect has long been recognized as necessary in the absence of specialized centers for the care of abused children, even if the patient does not warrant in-hospital care [7]. The investigation is time-consuming, with initial evaluations by a governmental agency (e.g. 
Department of Children and Family Services) lasting up to two or more days [8].

Previous studies, many from over a decade ago, have demonstrated that when comparing NAT to accidental trauma (AT), NAT was associated with a longer hospital length of stay (LOS), and a higher mortality rate [2, 9-14]. However, these studies are limited by the fact that many are comprised of either single-center or two-institution study populations, thus, limiting the generalizability of these results. Second, these studies were performed prior to changes in our healthcare and trauma systems that led to the development of multidisciplinary teams, as well as the certification and utilization of child abuse pediatric specialists [15-18]. Since the implementation of the Affordable Care Act, a migration from fee-for-service to performance-based payment has occurred. In addition, the use of quality metrics to compare hospitals, such as LOS have become widespread. Prolonged LOS due to NAT may cause the performance review of these centers to be negatively impacted $[19,20]$.

We sought to perform a contemporary analysis comparing NAT to AT in pediatric patients, hypothesizing that NAT is associated with longer hospital LOS compared to AT. This may identify a potential area for improvement aimed at reducing unnecessarily prolonged hospital LOS, as well as healthcare costs in these patients. Furthermore, it may illuminate a potential flaw in utilizing LOS as an outcome metric to gauge hospital performance and compare the quality of care between trauma centers.

\section{Methods}

A retrospective analysis of the Pediatric Trauma Quality Improvement Program (TQIP) database was performed between January 2014 and December 2016. The study was approved by the institutional review board at University of California, Irvine. All patients 1-16 years of age were included. Those presenting after NAT were compared to those sustaining AT. NAT was defined by the following event-codes: 967-967.9 (Table 4). The primary outcome was total hospital LOS. Secondary outcomes included: intensive care unit (ICU) LOS, ventilator days, in-hospital complications (i.e. deep vein thrombosis (DVT), pneumonia, and acute kidney injury (AKI)), and in-hospital mortality.

Patient variables collected included age, gender, the lowest systolic blood pressure (SBP) within 24-h of admission, massive-transfusion (defined by $\geq 6$ units of red blood cells (RBC) transfused within $4 \mathrm{~h}$ of arrival, injury severity score (ISS), and the abbreviated injury scale (AIS) for the head, thorax, abdomen and lower extremity. Descriptive statistics were performed for all variables. A Student's $t$ test or
Mann-Whitney $U$ test was used to compare continuous variables and Chi-square was used to compare categorical variables for bivariate analysis. Categorical data were reported as percentages, and continuous data was reported as medians with interquartile range or as means with standard deviation.

The median total hospital LOS was calculated for the entire dataset. A multivariable logistic regression model was then used to determine if NAT patients had risk for prolonged LOS compared to those with AT, while controlling for covariates including severe AIS of the head, thorax, abdomen, and lower extremity, ISS $\geq 25$, and pneumonia. This was reported with an odds ratio (OR) with $95 \%$ confidence intervals (CI). The analysis was performed based on the set of patients with complete data for all variables in the model. Differences with $p<0.05$ were considered statistically significant. All statistical analyses were performed with IBM SPSS Statistics for Windows, Version 24. (Armonk, NY: IBM Corp).

\section{Results}

\section{Patient demographics, characteristics, and primary outcome}

From 93,089 pediatric trauma patients, $417(<0.1 \%)$ sustained NAT. Compared to AT patients, NAT victims had a similar percentage of males $(60.2 \%$ vs. $64.1 \%, p=0.10)$ but a lower median age ( 3 vs. 9 years, $p<0.001$ ). Those with NAT had a higher SBP ( 88 vs. $79 \mathrm{~mm} \mathrm{Hg}, p=0.001$ ) and median ISS (10 vs. $5, p<0.001$ ) when compared to AT patients. Victims of NAT had a higher rate of high-grade injuries (AIS grade $>3)$ to the head $(38.1 \%$ vs. $8.5 \%, p<0.001)$ and abdomen $(6.2 \%$ vs. $2.4 \%, p<0.001)$. The NAT cohort had a similar percentage of high-grade injuries (AIS grade $>3$ ) to the thorax ( $2.2 \%$ vs. $1.6 \%, p=0.33)$ and lower extremity ( $0.5 \%$ vs. $0.4 \%, p=0.78)$ when compared to the AT cohort (Table 1). Compared to AT, the NAT cohort had a longer median LOS ( 4 vs. 2 days, $p<0.001$ ).

\section{Risk of increased length of stay}

The median LOS for all patients in the dataset was 2 days. In comparison to all patients involved in our pediatric trauma cohort, NAT was an independent risk factor for a LOS $\geq 2$ days (OR 4.99, 95\% CI 3.55-7.01, $p<0.001$ ) after controlling for ISS $\geq 25$, pneumonia, severe-AIS (AIS grade $>3$ ) of the head, thorax, abdomen, and lower extremity (Table 2). 
Table 1 Demographics of pediatric trauma patients with and without non-accidental trauma

\begin{tabular}{lllc}
\hline Characteristic & $\begin{array}{l}\text {-NAT } \\
(n=92,672)\end{array}$ & $\begin{array}{l}\text { +NAT } \\
(n=417)\end{array}$ & $p$ value \\
\hline Age, year, median (IQR) & $9.0(8)$ & $3.0(3)$ & $<0.001$ \\
Male, $n(\%)$ & $59,389(64.1 \%)$ & $251(60.2 \%)$ & 0.10 \\
ISS, median (IQR) & $5.0(5)$ & $10.0(16)$ & $<0.001$ \\
Lowest SBP within 24 h, & $79.0(85)$ & $88.0(21)$ & 0.001 \\
median (IQR) & & & \\
AIS (grade >3), $n(\%)$ & & $159(38.1 \%)$ & $<0.001$ \\
Head & $7890(8.5 \%)$ & $9(2.2 \%)$ & 0.33 \\
Thorax & $1445(1.6 \%)$ & $26(6.2 \%)$ & $<0.001$ \\
Abdomen & $2236(2.4 \%)$ & $2(0.5 \%)$ & 0.78 \\
Lower extremity & $364(0.4 \%)$ & \multicolumn{2}{c}{} \\
\hline
\end{tabular}

$N A T$ Non-accidental trauma, IQR interquartile range, ISS injury severity score, SBP systolic blood pressure, AIS abbreviated injury scale

Table 2 Multivariable logistic regression analysis for risk of increased length of stay ( $\geq 2$ days)

\begin{tabular}{lllr}
\hline Mechanism & OR & CI & $p$ value \\
\hline Non-accidental trauma & 4.99 & $3.55-7.01$ & $<0.001$ \\
Severe-AIS head & 2.96 & $2.78-3.16$ & 0.001 \\
Severe-AIS thorax & 3.26 & $2.73-3.88$ & $<0.001$ \\
Severe-AIS abdomen & 9.71 & $8.06-11.70$ & $<0.001$ \\
Severe-AIS lower extremity & 5.68 & $3.91-8.26$ & $<0.001$ \\
ISS $\geq 25$ & 2.65 & $2.32-3.03$ & $<0.001$ \\
Pneumonia & 55.03 & $7.67-394.54$ & $<0.001$ \\
\hline
\end{tabular}

AIS Abbreviated injury scale, ISS injury severity score

\section{Secondary outcomes and complications}

Compared to AT patients, those presenting after NAT had a longer median ICU LOS ( 3 vs. 2 days, $p<0.001$ ) and more ventilator days ( 4 vs. 2 days, $p<0.001$ ), required a lower median number of transfused RBC units within $4 \mathrm{~h}(1 \mathrm{vs}$. $1.7, p=0.04)$, had a higher rate of AKI $(1 \%$ vs. $<0.01 \%$, $p<0.001)$ but had similar rates of DVT $(0.5 \%$ vs. $0.1 \%$, $p=0.06)$, and pneumonia $(0.5 \%$ vs. $0.2 \%, p=0.33)$. NAT was associated with a higher mortality rate $(10.3 \%$ vs. $0.8 \%$, $p<0.001)$ and a lower rate of patients discharged to home, compared to AT (74.6\% vs. $95.9 \%, p=0.001)$ (Table 3 ).
Table 3 Clinical outcomes in pediatric patients with and without nonaccidental trauma

\begin{tabular}{lllc}
\hline Outcome & $\begin{array}{l}\text { NAT } \\
(n=92,672)\end{array}$ & $\begin{array}{l}\text { +NAT } \\
(n=417)\end{array}$ & $p$ value \\
\hline LOS, days, median (SD) & $2.0(2)$ & $4.0(8)$ & $<0.001$ \\
ICU, days, median (SD) & $2.0(2)$ & $3.0(5)$ & $<0.001$ \\
Ventilator, days, median & $2.0(4)$ & $4.0(5)$ & $<0.001$ \\
$\quad$ (SD) & & & \\
PRBC transfusion units & $1.7(2)$ & $1.0(1)$ & 0.04 \\
within 4 h, median & & & \\
$\quad$ IQR) & & & \\
Complications, $n(\%)$ & & $4(1.0 \%)$ & $<0.001$ \\
Acute kidney injury & $42(<0.01 \%)$ & $2(0.5 \%)$ & 0.06 \\
Deep vein thrombosis & $124(0.1 \%)$ & $2(0.5 \%)$ & 0.33 \\
Pneumonia & $227(0.2 \%)$ & $311(74.6 \%)$ & 0.001 \\
Discharge home, $n(\%)$ & $889,092(95.9 \%)$ & & \\
Mortality, $n(\%)$ & $703(0.8 \%)$ & $43(10.3 \%)$ & $<0.001$ \\
\hline
\end{tabular}

NAT Non-accidental trauma, LOS length of stay, IQR interquartile range, $I C U$ intensive care unit, $P R B C$ packed red blood cells, ARDS acute respiratory distress syndrome

\section{Discussion}

We performed a review of a large trauma database, comparing patient characteristics, injury-related properties, and outcomes between pediatric NAT and AT victims. We reported that NAT victims had more severe injuries, longer hospital and ICU LOS, and higher mortality rates when compared to AT victims. These findings support previous studies reporting that NAT was associated with greater injury severity and higher mortality rates [9-13]. However, we are the first national study in more than a decade to report that NAT victims were nearly five times more likely to require a LOS of $\geq 2$ days, independent of injury severity. The results of our study suggest there may be a need to investigate reasons for prolonged hospital stays in this patient population, to determine if NAT investigations may be a contributing factor to unwarranted hospitalizations, and if so, to potentially question the use of LOS metrics in NAT patients when evaluating the quality of care delivered by a hospital.

A 10-year review of the National Pediatric Trauma Registry from 1988 to 1997 by DiScala et al. identified more than 18,000 pediatric trauma patients and reported that NAT victims $(n=1997)$ had LOS of 9.8 days compared to 3.8 days for AT $(n=16,831)$ [12]. Nearly a decade later, using the national Kids' Inpatient Database (KID) (2003-2006), Lane et al. identified more than 4000 pediatric victims of abdominal trauma and found NAT to the abdomen $(n=234)$ to be associated with longer hospitalization ( 7.9 vs. 6.4 days, 
$p<0.01)$ and higher hospital charges $(\$ 24,343$ vs. $\$ 19,341)$ compared to AT $(n=4200)$ [9]. Developments such as the introduction of multidisciplinary teams, as well as the certification and utilization of child abuse pediatric specialists, were intended to enhance decision making in the best interest of the abused child and to improve outcomes such as cost and LOS [15-18]. Although we demonstrated shorter LOS among NAT victims compared to previous studies, the LOS for NAT victims remains significantly longer compared to AT patients.

We reported a NAT mortality rate of $10.3 \%$, which is the highest to be reported in nearly two decades, and demonstrated that NAT victims are 13-times more likely to die in the hospital compared to children who sustained AT [12]. The increased mortality of NAT compared to AT may be partially explained by the association of higher injury severity, in particular, high-grade injuries to the head and abdomen. Additionally, victims of NAT were younger (3 vs. 9 years old) than children who sustained AT. Previous authors have suggested that the young age of NAT victims may contribute to increased mortality as they are unable to protect themselves, escape, or report abuse [10, 12]. Our findings are similar to previous studies which reported that pediatric NAT victims were younger and had higher mortality than AT victims [2, 9-13]. Our findings affirm that NAT is a continued public health epidemic, and requires further intervention.

A previous single-center retrospective study performed by Lee et al. evaluated the hospital costs associated with discharge delays in children hospitalized for abuse and neglect [21]. A majority of medically cleared NAT victims with discharge delays were in CPS custody and did not necessarily warrant inpatient hospitalization. Although we were unable to identify NAT victims who stayed beyond medical clearance in our study, the increased LOS independent of injury severity in NAT compared to AT suggests NAT victims experienced discharge delays for reasons not influenced by the quality of trauma care provided. Similar to the study by Lee et al. these victims likely experienced discharge delays due to the time required for child abuse investigations and appropriate placement. These findings demonstrate there is a need to develop alternative caregiving arrangements by staff other than physicians and nurses, especially in the inpatient hospital setting, who can provide a safe environment at a lower cost while a medically cleared NAT child is awaiting placement. Future studies should evaluate the feasibility and cost effectiveness of this type of caregiving arrangement, including out-of-hospital facilities or specific departments within the hospital. Other methods that may reduce NAT altogether, include increasing funding for child abuse prevention programs, foster care, and child protective services. Multiple studies have demonstrated that child abuse prevention programs resulted in significant subsequent reductions in NAT rates, which may be associated with lowering overall healthcare costs [22-27]. For instance, Foster et al. estimated the costs associated with a population-wide parenting program aimed at reducing child mistreatment, could be recovered in a single year, with just a $10 \%$ reduction in the rate of overall child maltreatment [27]. Increasing funding to foster care programs may represent another cost-effective approach to reducing the healthcare costs associated with hospital LOS in victims of NAT. In fact, the Children and Youth Services Review recently found that foster care reimbursement rates are far lower than would be expected to meet children's basic needs [28]. The low reimbursement rates may be contributing to the increased LOS of NAT by reducing the availability of foster homes for immediate placement of these victims.

There are a number of limitations to this study, including those inherent to a retrospective database such as input bias and missing data. Pertinent data variables missing from the database include hospital charges, hospital LOS after medical clearance, prior admissions for NAT, socioeconomic factors, time to identifying NAT and obtaining a child abuse specialist and/or CPS consult, as well as time from consult to the time of final determination of the child's disposition. Furthermore, our analysis is limited by the relatively small size of NAT victims $(n=417)$ compared to all pediatric trauma patients $(n=93,089)$ in our study population. There are a few factors that may have contributed to the limited number of NAT victims. Specifically, our study only includes NAT victims that presented to one of the approximately 125 trauma centers enrolled in the Pediatric TQIP. Additionally, inclusion in the pediatric TQIP database requires victims to present as a trauma admission and have a AIS > 1, signifying that only those with significant trauma are included within the database. Therefore, victims who did not warrant evaluation and admission as a trauma patient or were later discovered to have minor traumatic injuries were not included in this study. The study is further limited by the absence of a hospital cost analysis providing more information about potential differences in hospital charges of NAT compared to AT. Additionally, definitive diagnosis of NAT and AT can be challenging since it is often based on clinical suspicion. Therefore, it is possible that some patients identified as AT were actually victims of NAT. Despite these limitations, our study adds to the existing body of literature on pediatric NAT, a continued national crisis associated 
with high rates of mortality and increased LOS and calls to action further research and injury prevention efforts for this epidemic.

\section{Conclusions}

Our national database study demonstrated that pediatric victims of NAT were more severely injured and had higher mortality rates compared to children sustaining AT. We reported NAT was associated with an increased LOS when compared to AT, independent of injury severity. Future studies should explore the factors contributing to longer hospitalizations in this patient population, as well as determine the incidence of patients who were medically cleared but awaiting NAT work-up before they could be discharged. In addition, the feasibility of alternative caregiving arrangements to the inpatient hospitalization of victims of NAT who are medically cleared but awaiting a safe disposition merits evaluation. Finally, when evaluating quality of care metrics such as mortality and LOS, NAT should be considered, as this may unfairly adversely affect centers seeing and/or identifying a higher volume of NAT patients.

\section{Compliance with ethical standards}

Conflict of interest The authors declare that they have no conflict of interest.

Ethical approval This article does not contain any studies with human participants or animals performed by any of the authors.

\section{Appendix}

See Table 4.

Table 4 ICD-9 Event Codes for non-accidental trauma

ICD-9 event codes

\begin{tabular}{ll}
\hline 967 & Child/adult abuse by father/stepfather/male partner \\
967.1 & Child/adult abuse by other specified person \\
967.2 & Child/adult abuse by mother/stepmother/female partner \\
967.3 & Child/adult abuse by spouse/partner/ex-spouse/ex-partner \\
967.4 & Child/adult abuse by child \\
967.5 & Child/adult abuse by sibling \\
967.6 & Child/adult abuse by grandparent \\
967.7 & Child/adult abuse by other relative \\
967.8 & Child/adult abuse by non-related caregiver \\
967.9 & Child/adult abuse by unspecified person
\end{tabular}

\section{References}

1. U.S. Department of Health and Human Services, Administration for Children and Families, Administration on Children, Youth and Families, Children's Bureau (2016) Child maltreatment 2016. https://www.acf.hhs.gov/cb/research-data-technology/ statistics-research/child-maltreatment. Accessed 16 Aug 2018

2. Ward A, Iocono JA, Brown S, Ashley P, Draus JM (2015) Nonaccidental trauma injury patterns and outcomes: a single institutional experience. Am Surgeon 81(9):835-838

3. Kim PT, Falcone RA (2017) Nonaccidental trauma in pediatric surgery. Surg Clinics 97(1):21-33

4. Gooding C (2017) Nonaccidental trauma and the pediatric surgical patient: practice considerations. J Pediatr Surg Nurs $6(2): 34-38$

5. Lopez ON, Hughes BD, Adhikari D, Williams K, Radhakrishnan RS, Bowen-Jallow KA (2018) Sociodemographic determinants of non-accidental traumatic injuries in children. Am J Surg 215(6):1037-1041

6. Sola R Jr, Waddell VA, Peter SDS, Aguayo P, Juang D (2018) Non-accidental trauma: a national survey on management. Injury 49(5):921-926

7. Shira JE, Diamond J, O'Connor ME, Packard J, Reynolds M, Schaeffer HA, VanOstenberg PR (1998) Medical necessity for the hospitalization of the abused and neglected child. Pediatrics 101(4):715-716

8. Sullivan CM (2011) Child abuse and the legal system: the orthopaedic surgeon's role in diagnosis. Clin Orthop Relat Res 469(3):768-775

9. Lane WG, Lotwin I, Dubowitz H, Langenberg P, Dischinger P (2011) Outcomes for children hospitalized with abusive vs non-inflicted abdominal trauma. Pediatrics 127(6):e1400

10. Roaten JB, Partrick DA, Nydam TL, Bensard DD, Hendrickson RJ, Sirotnak AP, Karrer FM (2006) Nonaccidental trauma is a major cause of morbidity and mortality among patients at a regional level 1 pediatric trauma center. J Pediatr Surg 41(12):2013-2015

11. Estroff JM, Foglia RP, Fuchs JR (2015) A comparison of accidental and nonaccidental trauma: it is worse than you think. $\mathbf{J}$ Emerg Med 48(3):274-279

12. DiScala C, Sege R, Li G, Reece RM (2000) Child abuse and unintentional injuries: a 10-year retrospective. Arch Pediatr Adolesc Med 154(1):16-22

13. Larimer EL, Fallon SC, Westfall J, Frost M, Wesson DE, NaikMathuria BJ (2013) The importance of surgeon involvement in the evaluation of non-accidental trauma patients. J Pediatr Surg 48(6): 1357-1362

14. Ortega HW, Vander Velden H, Kreykes NS, Reid S (2013) Childhood death attributable to trauma: is there a difference between accidental and abusive fatal injuries? J Emerg Med 45(3):332-337

15. Draus JM (2017) A multidisciplinary child protection team improves the care of nonaccidental trauma patients. Am Surgeon 83(5):477-481

16. Wallace GH, Makoroff KL, Malott HA, Shapiro RA (2007) Hospital-based multidisciplinary teams can prevent unnecessary child abuse reports and out-of-home placements. Child Abuse Negl 31(6):623-629

17. Jones JG, Worthington T, Hawks F, Mercer SO, Jones BW, Woon L (1998) Ad hoc conferences of hospital and community professionals in cases of hospitalized physically abused children. Child Abuse Negl 22(1):63-68

18. Block RW, Palusci VJ (2006) Child abuse pediatrics: a new pediatric subspecialty. J Pediatr 148(6):711-712 
19. Protection P, Act AC (2010) Patient protection and affordable care act. Public Law 111(48):759-762

20. English WJ (2008) Rewarding provider performance: aligning incentives in medicare. Ann Intern Med 148(8):636

21. Lee M, Bachim A, Smith C, Camp EA, Donaruma-Kwoh M, Patel B (2017) Hospital costs and charges of discharge delays in children hospitalized for abuse and neglect. Hospital Pediatr 7(10):572-578

22. Prinz RJ, Sanders MR, Shapiro CJ, Whitaker DJ, Lutzker JR (2009) Population-based prevention of child maltreatment: the US Triple P system population trial. Prev Sci 10(1):1-12

23. Geeraert L, Van den Noortgate W, Grietens H, Onghena P (2004) The effects of early prevention programs for families with young children at risk for physical child abuse and neglect: a meta-analysis. Child Maltreatment 9(3):277-291

24. Barth RP (2009) Preventing child abuse and neglect with parent training: evidence and opportunities. The Future of Children 19(2):95-118
25. Mikton C, Butchart A (2009) Child maltreatment prevention: a systematic review of reviews. Bull World Health Organ $87: 353-361$

26. Irazuzta J, McJunkin JE, Danadian K, Arnold F, Zhang J (1997) Outcome and cost of child abuse. Child Abuse Negl 21(8):751-757

27. Foster EM, Prinz RJ, Sanders MR, Shapiro CJ (2008) The costs of a public health infrastructure for delivering parenting and family support. Children Youth Serv Rev 30(5):493-501

28. McLaughlin M, Jonson-Reid M (2017) The relationship between child welfare financing, screening, and substantiation. Children Youth Serv Rev 82:407-412

Publisher's Note Springer Nature remains neutral with regard to jurisdictional claims in published maps and institutional affiliations. 